\author{
Supporting Information
}

\title{
A Benzopyronin-based Two-photon Fluorescent Probe for Ratiometric Imaging of Lysosomal Bisulfite with Complete Spectral Separation
}

Umme Tamima, ${ }^{\dagger}$ Mithun Santra, ${ }^{* *}$ Chang Wook Song,${ }^{\dagger}$ Ye Jin Reo,${ }^{\dagger}$ and Kyo Han Ahn ${ }^{\dagger *}$

${ }^{\dagger}$ Department of Chemistry, Pohang University of Science and Technology (POSTECH), 77 Cheongam-Ro, Nam-Gu, Pohang, Gyungbuk 37673, Republic of Korea.

EaStCHEM School of Chemistry, University of Edinburgh, Joseph Black Building, King's Buildings, David Brewster Road, EH9 3FJ Edinburgh, UK.

*Email: msantra@ed.ac.uk (M. Santra); ahn@postech.ac.kr (K. H. Ahn)

\section{Contents}

1. Supporting figures

2. Supporting tables

3. NMR $\left({ }^{1} \mathrm{H} \&{ }^{13} \mathrm{C}\right)$ spectra

4. MASS spectra 


\section{Supporting figures}
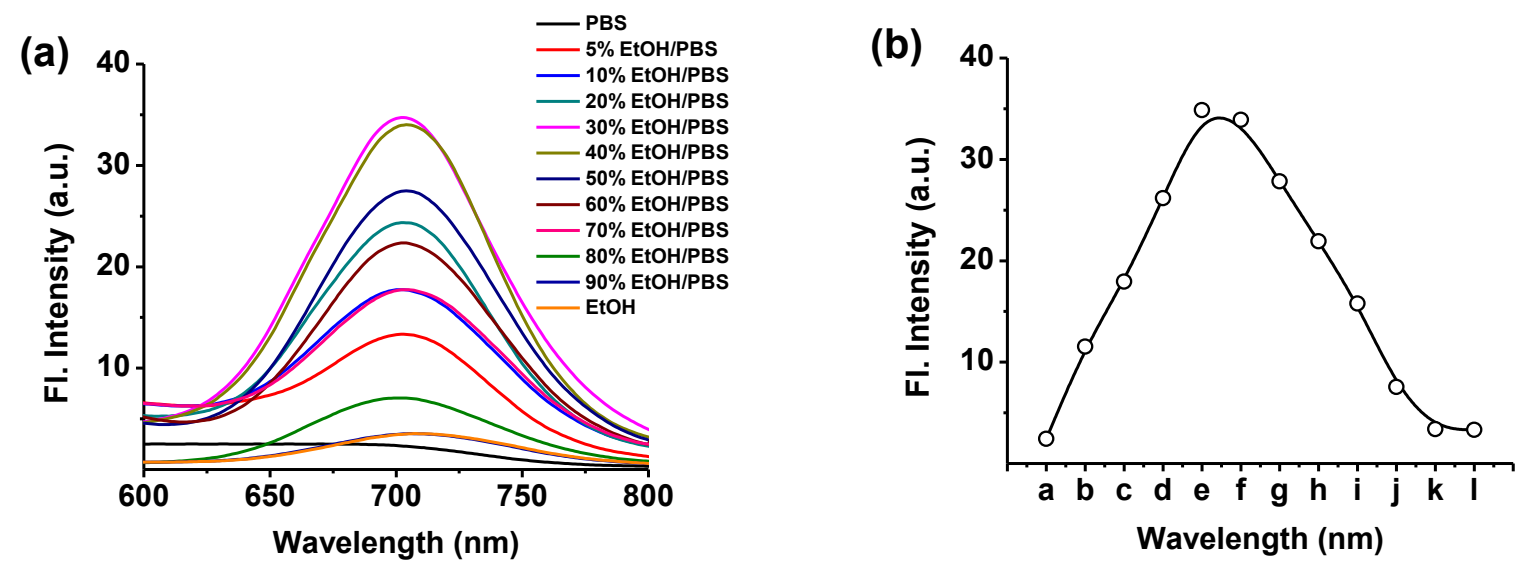

Figure S1. (a) Fluorescence spectral changes and (b) fluorescence intensity changes of BPyn $(10 \mu \mathrm{M})$ in various solvents at $\mathrm{pH}$ 5. $\lambda_{\mathrm{ex}}=480 \mathrm{~nm}$.

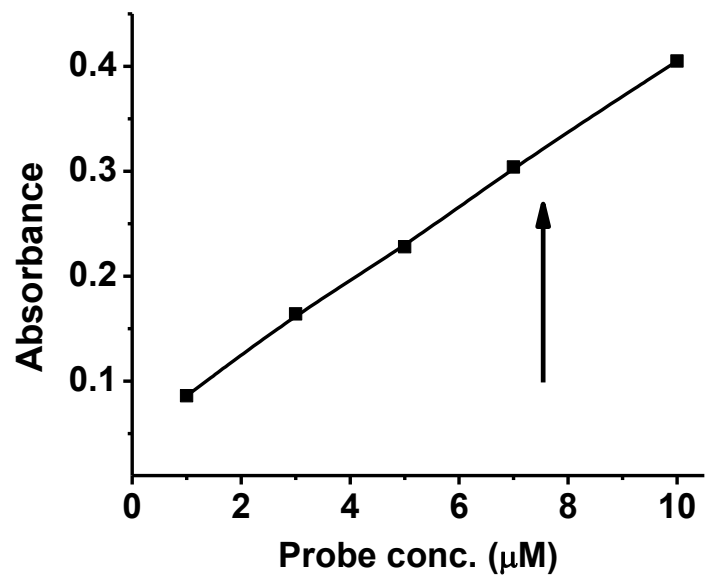

Figure S2. Absorbance changes of BPyn dependent on its concentration in PBS (pH 5) containing $30 \% \mathrm{EtOH}$ as a co-solvent, recorded at $25^{\circ} \mathrm{C}$.

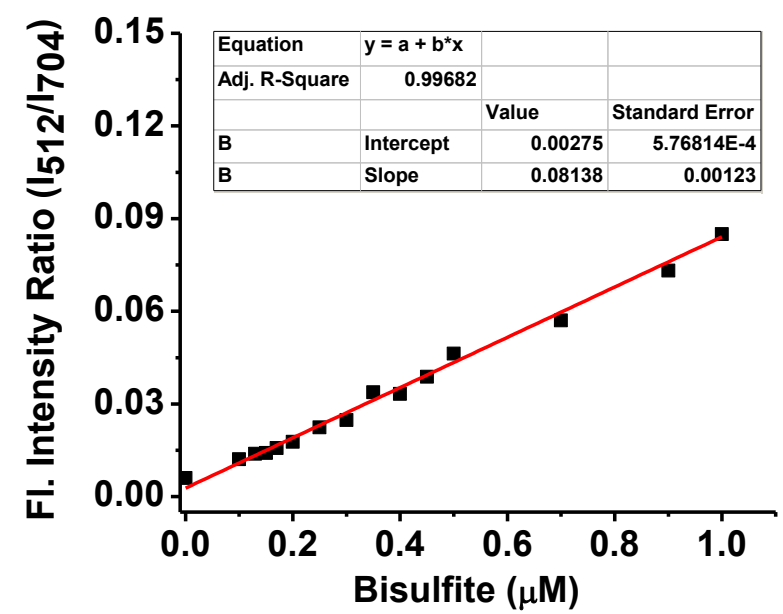


Figure S3. The emission intensity ratio $\left(I_{512 \mathrm{~nm}} / I_{704} \mathrm{~nm}\right)$ changes of BPyn $(10 \mu \mathrm{M})$ depending on [bisulfite] in a low concentration region $(0-1.0 \mu \mathrm{M})$ in PBS $(\mathrm{pH} 5)$ containing $30 \% \mathrm{EtOH}$ as a cosolvent. The spectra were recorded at $25{ }^{\circ} \mathrm{C}$ under excitation at $480 \mathrm{~nm}$. On the basis of this plot, the detection limit was calculated by following the equation,

Detection limit $(\mathrm{LOD})=3 \sigma / \mathrm{k}=0.09 \mu \mathrm{M}$,

Where $\sigma$ is the standard deviation of three blank measurements $=0.00239, k$ is the slope of the linear plot of the fluorescence intensity ratios changes $\left(I_{512 \mathrm{~nm}} / I_{704} \mathrm{~nm}\right)$ in the lower bisulfite concentration region $(0-1.0 \mu \mathrm{M})=0.08138$.

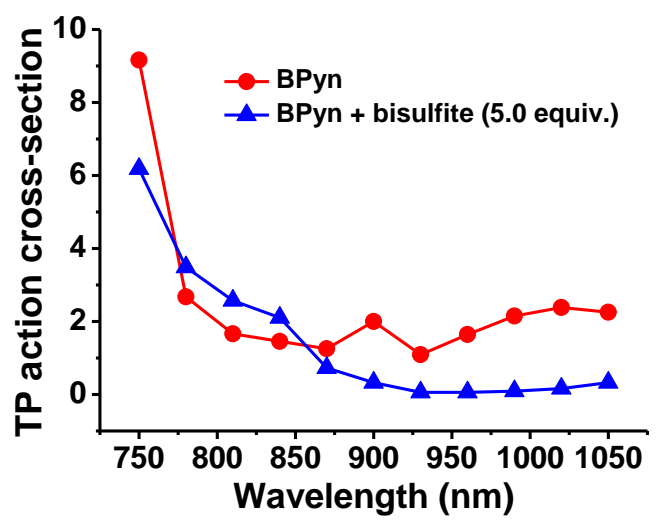

Figure S4. Two-photon action cross-section spectra of BPyn $(100 \mu \mathrm{M})$ and its bisulfite adduct (5.0 equiv.), measured in dioxane using rhodamine $\mathrm{B}$ (in EtOH) as a reference dye. The TP action crosssection values are obtained under approximations: Because we were able to collect emissions up to $665 \mathrm{~nm}$ using a commercial TPM, although the probe emits even over $800 \mathrm{~nm}$. To compensate this inherent limitation, we used a conversion factor (the whole emission spectral area integrated/the emission intensity integrated partially up to $665 \mathrm{~nm}$ from the blue side) to obtain approximate twophoton excited fluorescence intensity of probe.

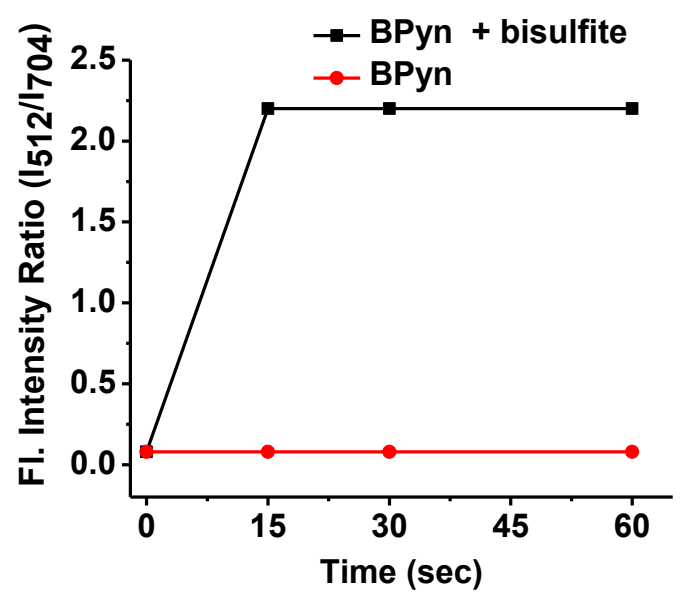

Figure S5. Time-dependent ratio intensities $\left(I_{512} / I_{704}\right)$ of BPyn $(10 \mu \mathrm{M})$ in the absence or presence of bisulfite $(20 \mu \mathrm{M})$ in PBS (pH 5) containing $30 \% \mathrm{EtOH}$ as a co-solvent. $\lambda_{\mathrm{ex}}=480 \mathrm{~nm}$. 


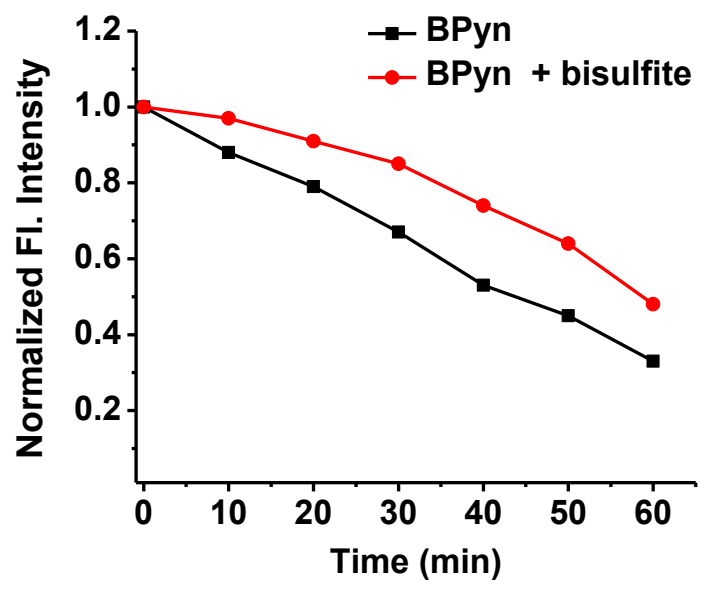

Figure S6. Photostablity of BPyn $(10 \mu \mathrm{M})$ in the absence and presence of bisulfite in PBS (pH 5) containing $30 \% \mathrm{EtOH}$ as a co-solvent, obtained under one-photon excitation at $\lambda_{\mathrm{ex}}=480 \mathrm{~nm}$.

(a)

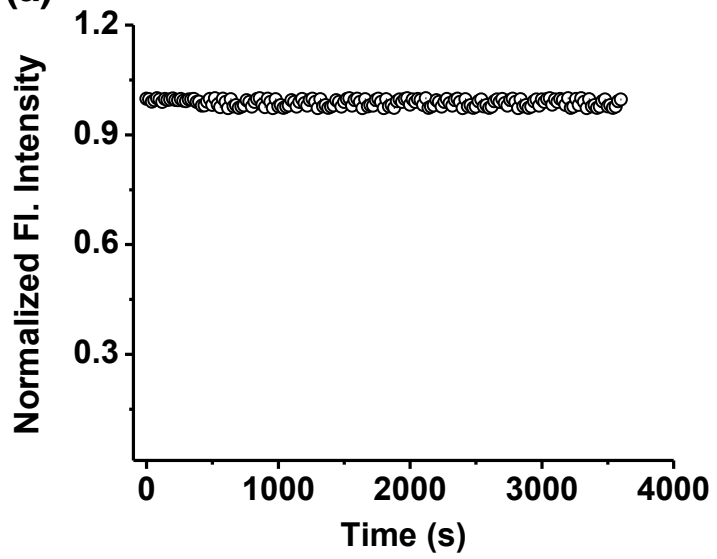

(b)

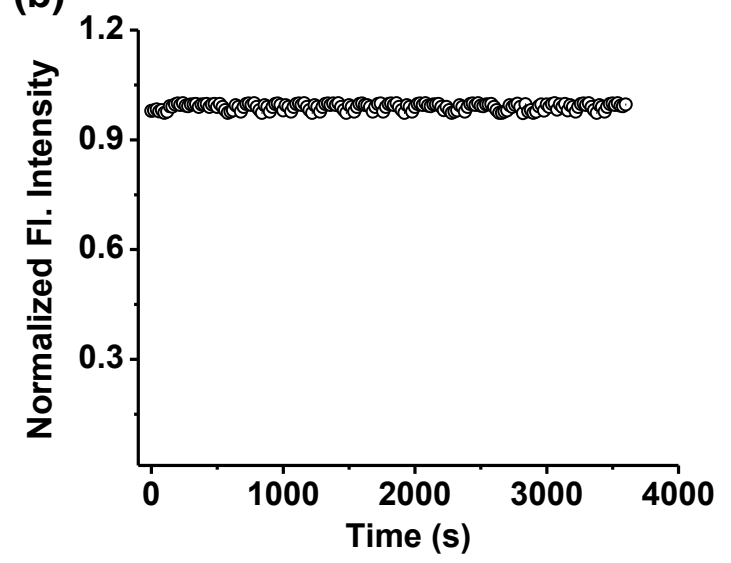

Figure S7. (a) Normalized fluorescence intensity of the probe and (b) its bisulfite adduct (10 $\mu \mathrm{M}$ in DMSO) under two-photon irradiation $\left(\lambda_{\mathrm{ex}}=900 \mathrm{~nm}, 50 \mathrm{~mW}\right)$; emission was collected in the range of $400-665 \mathrm{~nm}$. 

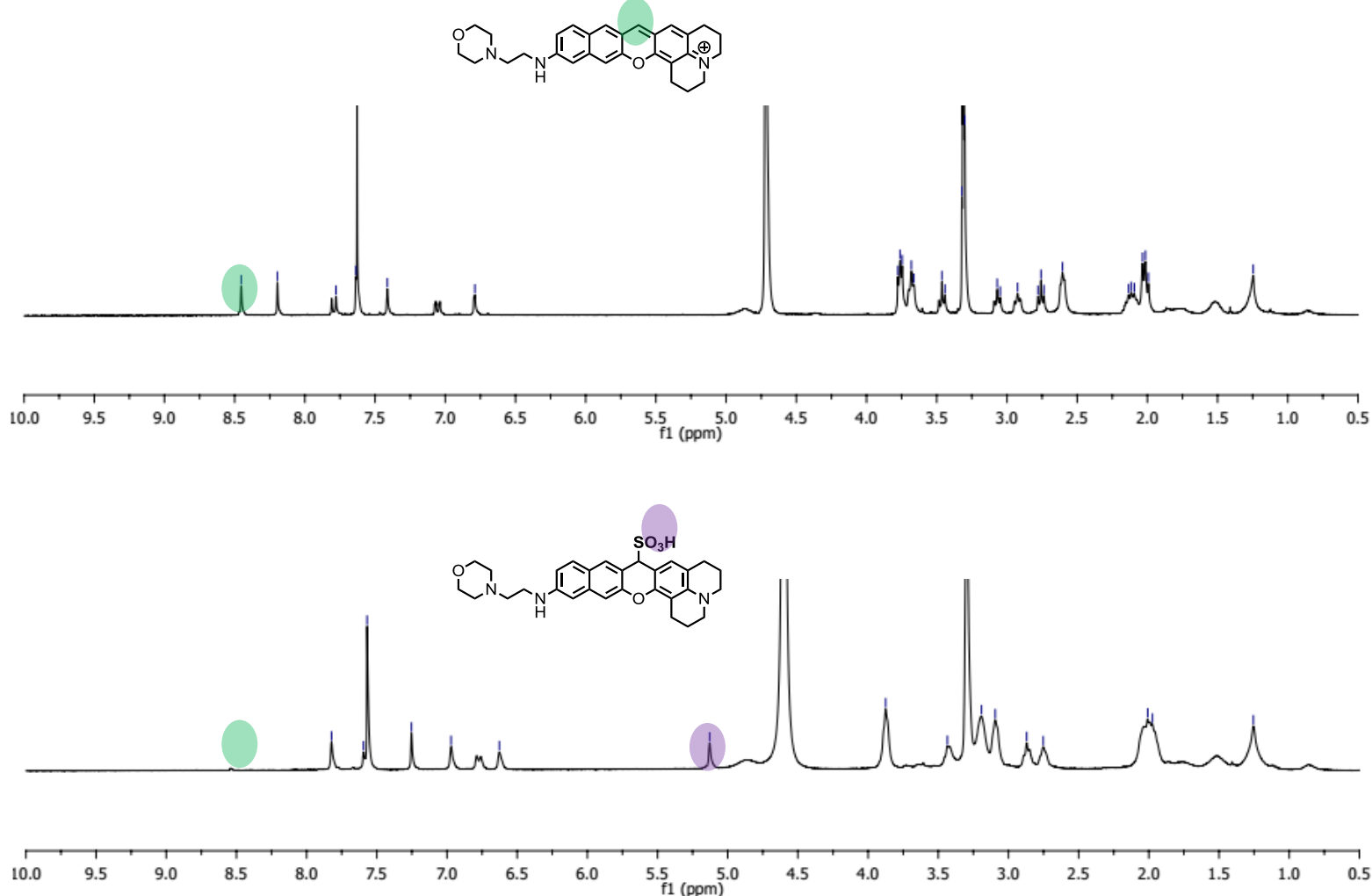

Figure S8. ${ }^{1} \mathrm{H}$ NMR spectra of BPyn alone (top) and BPyn in the presence of 5 equiv. of bisulfite (bottom) in $\mathrm{MeOD} / \mathrm{CDCl}_{3}(\sim 7 / 3)$ and $\mathrm{MeOD} / \mathrm{CDCl}_{3} / \mathrm{D}_{2} \mathrm{O}(\sim 3 / 6 / 1)$, respectively. 


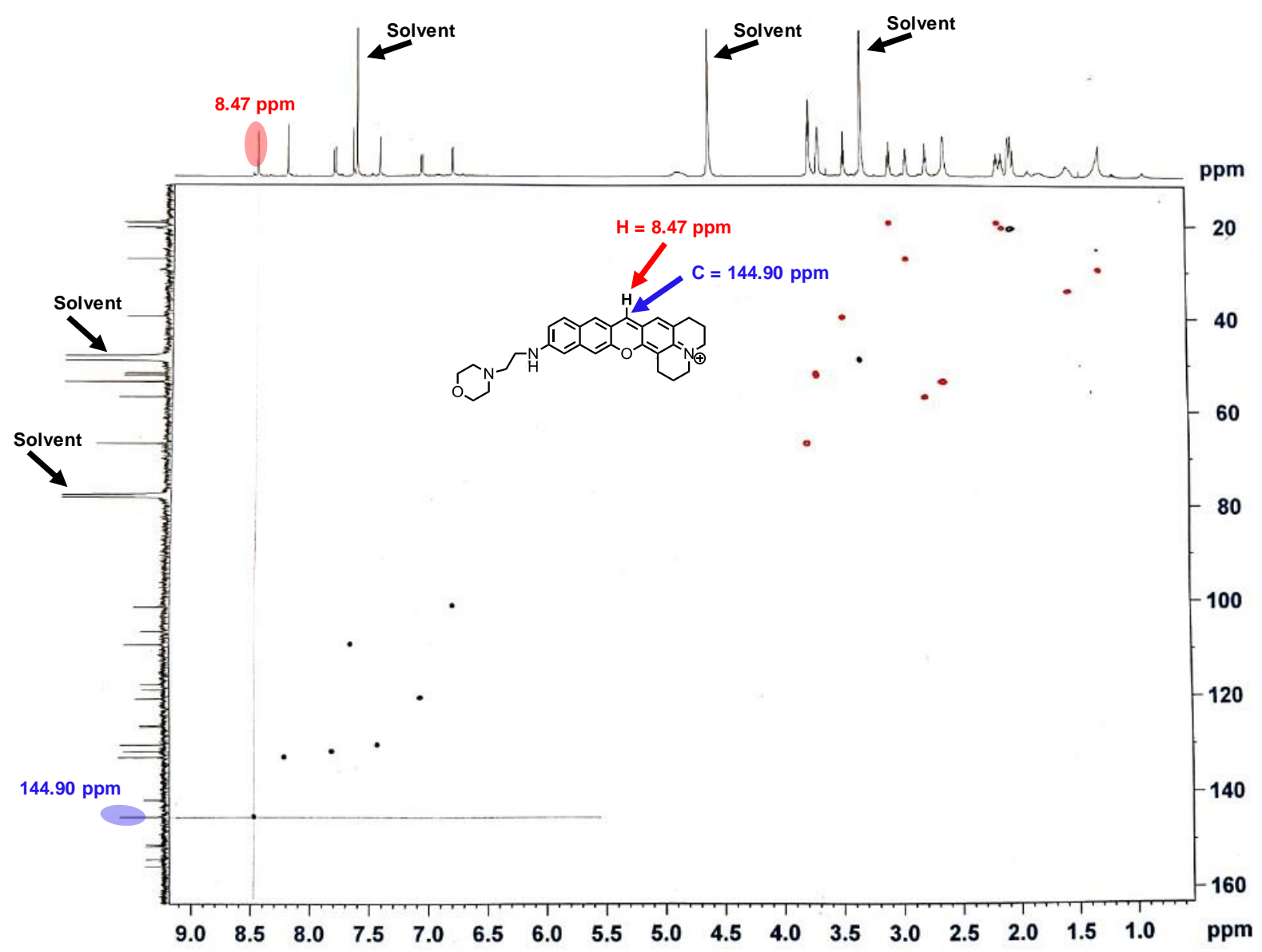

Figure S9. ${ }^{1} \mathrm{H}-{ }^{13} \mathrm{C}$ HSQC spectrum of BPyn in $\mathrm{MeOD} / \mathrm{CDCl}_{3}(\sim 7 / 3)$. 


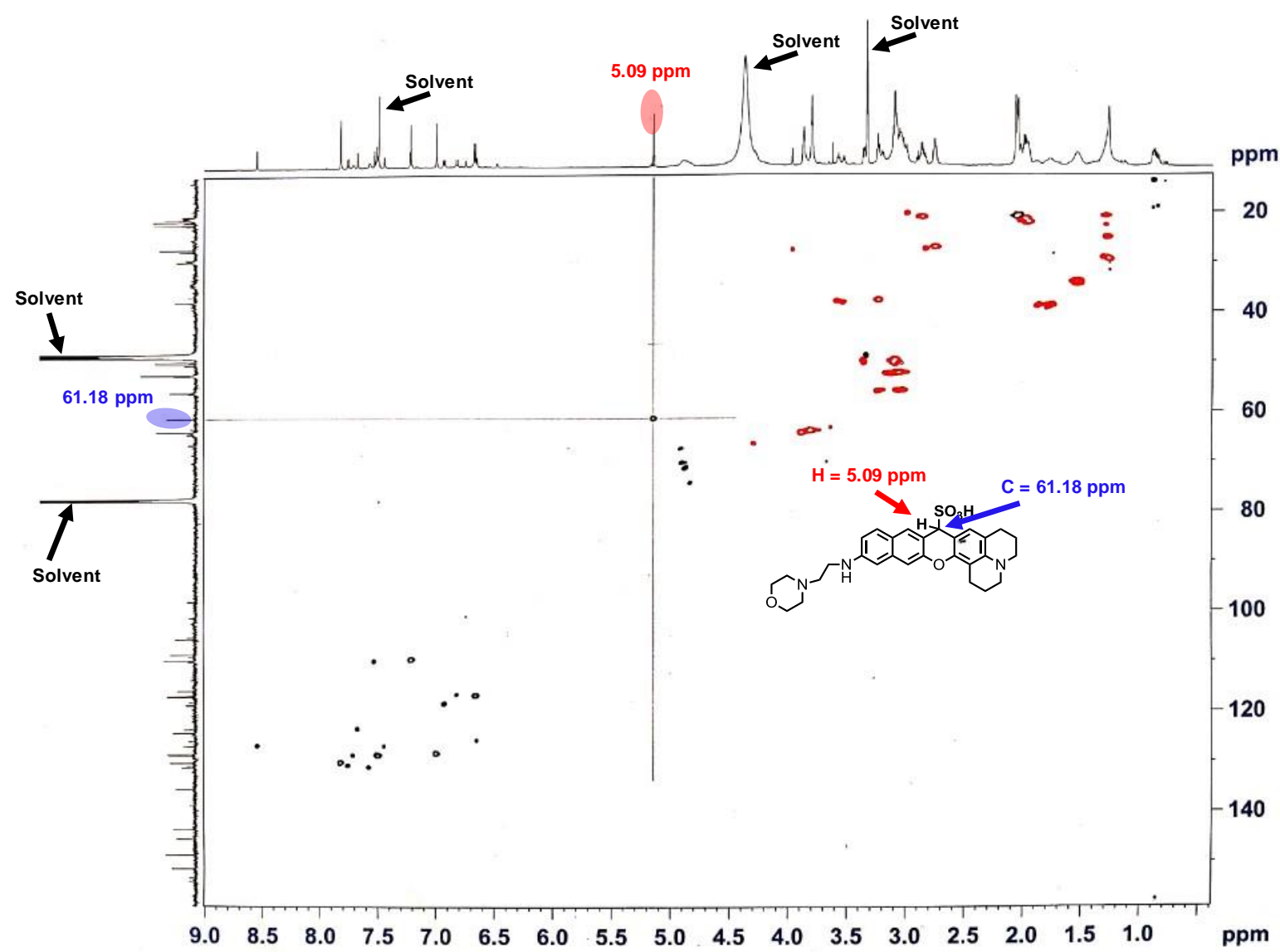

Figure S10. ${ }^{1} \mathrm{H}^{13} \mathrm{C}$ HSQC spectrum of BPyn in the presence of 5.0 equiv. of bisulfite in $\mathrm{MeOD} / \mathrm{CDCl}_{3} / \mathrm{D}_{2} \mathrm{O}(\sim 3 / 6 / 1)$, respectively. 


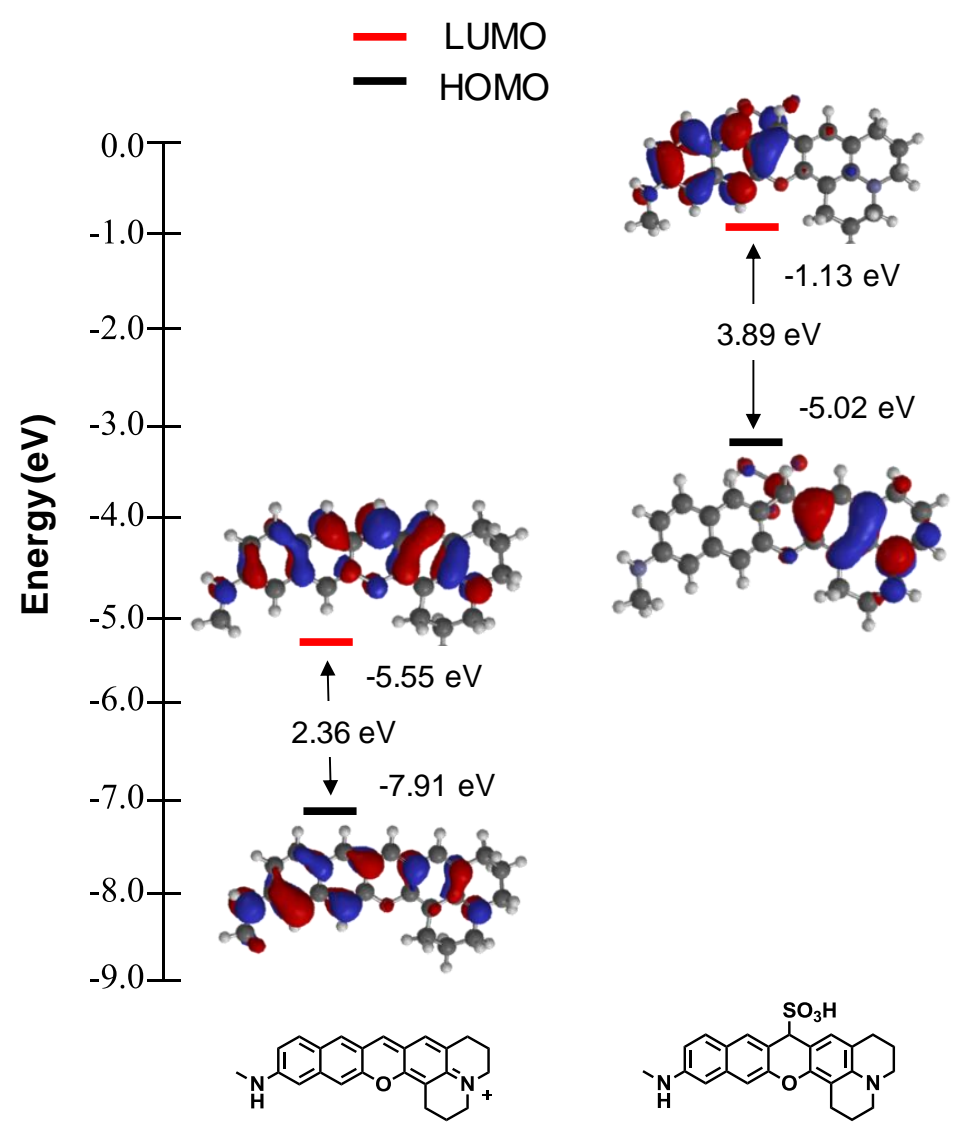

Figure S11. Frontier molecular orbitals and corresponding energy levels of simplified structures of the probe ( $N$-methylamino group instead of the morpholine donor) and its bisulfite adduct.

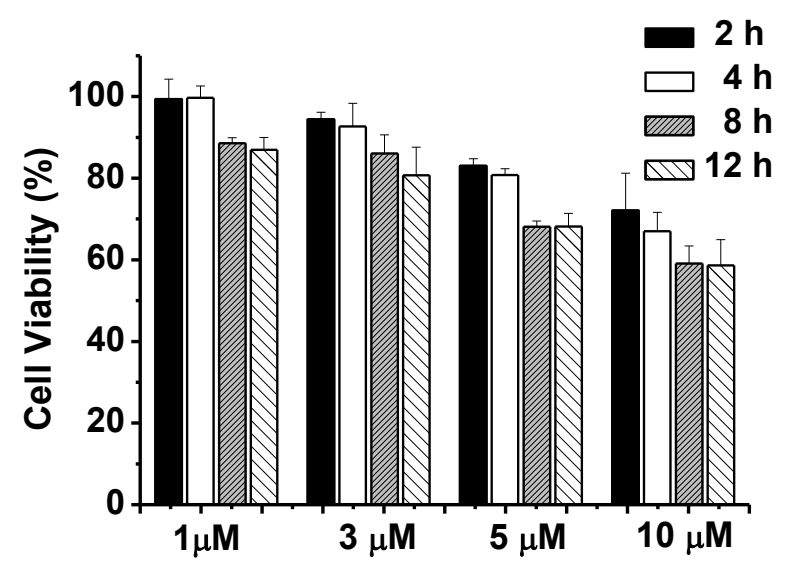

Figure S12. Viability of HeLa cells incubated for $12 \mathrm{~h}$ with varying concentrations $(0-10 \mu \mathrm{M})$ of BPyn. 


\section{Supporting tables}

Table S1. Photophysical properties of BPyn and its bisulfite adduct.

\begin{tabular}{|l|l|l|l|l|l|}
\hline Compounds & $\begin{array}{l}\lambda_{\mathrm{abs}} \\
(\mathrm{nm})^{a}\end{array}$ & $\begin{array}{l}\varepsilon\left(\mathrm{L} \mathrm{mol}^{-1}\right. \\
\left.\mathrm{cm}^{-1}\right)^{b}\end{array}$ & $\begin{array}{l}\lambda_{\mathrm{em}} \\
(\mathrm{nm})^{c}\end{array}$ & $\begin{array}{l}\Phi_{\mathrm{F}}{ }^{d} \\
\text { Brightness } \\
\left(\varepsilon \times \Phi_{\mathrm{F}}\right)\end{array}$ \\
\hline BPyn & 613 & 14,500 & 704 & 0.08 & 1,200 \\
\hline BPyn-SO3H & 426 & 22,400 & 512 & 0.12 & 2,700 \\
\hline
\end{tabular}

${ }^{a}$ The maximum one-photon absorption wavelength $\left(\lambda_{\mathrm{abs}}\right) .{ }^{\mathrm{b}}$ Molar extinction coefficient $(\varepsilon)$. ${ }^{\mathrm{c}}$ The maximum one-photon emission wavelength $\left(\lambda_{\mathrm{em}}\right)$. All measured in $30 \% \mathrm{EtOH} / \mathrm{PBS}$ buffer $(\mathrm{pH}=5.0)$. ${ }^{\mathrm{d}}$ Fluorescence quantum yields $\left(\Phi_{\mathrm{F}}\right)$ measured in ethanol using Nile blue $\left(\Phi_{\mathrm{F}}=0.27\right.$ in EtOH $)$ as reference. 
Table S2. Summary of known two-photon probes for bisulfite.

\begin{tabular}{|c|c|c|c|c|c|c|c|c|}
\hline $\begin{array}{l}\text { Probe and } \\
\text { references }\end{array}$ & $\begin{array}{l}\text { Signal- } \\
\text { ing } \\
\text { mode }\end{array}$ & $\begin{array}{l}\lambda_{\text {ex }} \\
\mathrm{nm}^{\mathrm{a}}\end{array}$ & $\begin{array}{l}\lambda_{\mathrm{em}} \\
\mathrm{nm}^{\mathrm{b}}\end{array}$ & $\begin{array}{l}\text { Organelle } \\
\text { targeting }\end{array}$ & $\begin{array}{l}\text { Detection } \\
\text { medium }\end{array}$ & $\begin{array}{l}\text { Detec- } \\
\text { tion } \\
\text { time }\end{array}$ & $\begin{array}{l}\text { Detection } \\
\text { model }\end{array}$ & $\begin{array}{l}\text { Interference } \\
\text { with bio- } \\
\text { thiols }\end{array}$ \\
\hline $\begin{array}{l}\text { Chem. B, } \\
\text { J. Mater. } \\
\text { 2016, } 4,7888 \text { - } \\
-7894\end{array}$ & Turn-on & 490 & 600 & No & $\begin{array}{c}\text { aq. } \\
\text { Buffer:DMSO } \\
(98: 2, \mathrm{v} / \mathrm{v}) \\
\text { using } 200 \mathrm{mM} \\
\mathrm{Na}_{2} \mathrm{HPO} 4: \mathrm{citri} \\
\mathrm{c} \text { acid buffer } \\
\text { solution at pH } \\
5.0\end{array}$ & $10 \mathrm{~min}$ & $\begin{array}{l}\text { HeLa } \\
\text { cells, } \\
\text { Tissue }\end{array}$ & $\begin{array}{c}\text { Only response } \\
\text { to } \\
\mathrm{HSO}_{3}{ }^{-} / \mathrm{SO}_{3}{ }^{2-}\end{array}$ \\
\hline $\begin{array}{l}\text { Densors and } \\
\text { Actuators B, } \\
\mathbf{2 0 1 6}, \quad 235 \text {, } \\
362-369\end{array}$ & Turn-on & 345 & 420 & No & $\begin{array}{l}0.2 \mathrm{M} \\
\mathrm{Na}_{2} \mathrm{HPO}_{4} \\
\text { citric acid (pH } \\
5.0) \quad \text { aqueous } \\
\text { buffer, } \quad 1 \% \\
\text { DMSO }\end{array}$ & $5 \mathrm{~min}$ & $\begin{array}{l}\text { HeLa } \\
\text { cells }\end{array}$ & $\begin{array}{l}\text { No response } \\
\text { to } \mathrm{H}_{2} \mathrm{~S} \text {, } \mathrm{Cys} \text {, } \\
\mathrm{HCy}, \quad \mathrm{CN} ; \\
\mathrm{GSH}, \\
\text { checked; not } \\
\text { response to } \\
\mathrm{HSO}_{3}{ }^{-}\end{array}$ \\
\hline $\begin{array}{l}\text { Anal. Chem. } \\
\text { 2017, } 89 \text {, } \\
9388-9393\end{array}$ & 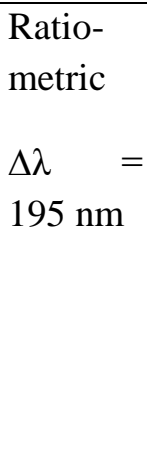 & 380 & $\begin{array}{l}450, \\
645\end{array}$ & $\begin{array}{l}\text { Mitochon } \\
\text {-dria }\end{array}$ & $\begin{array}{l}\text { PBS (pH 7.4), } \\
5 \% \text { DMSO }\end{array}$ & $\begin{array}{l}\text { Within } \\
\text { sec }\end{array}$ & $\begin{array}{l}\text { HeLa } \\
\text { cells, } \\
\text { Zebrafish }\end{array}$ & $\begin{array}{l}\text { No response } \\
\text { to } \mathrm{H}_{2} \mathrm{~S} \text {, Cys, } \\
\mathrm{Hcy} \text { GSH; } \\
\text { response to } \\
\mathrm{HSO}_{3}{ }^{-} / \mathrm{SO}_{3}{ }^{2-}\end{array}$ \\
\hline
\end{tabular}




\begin{tabular}{|c|c|c|c|c|c|c|c|c|}
\hline $\begin{array}{l}\text { Sensors and } \\
\text { Actuators B } \\
\text { 1228-1237 }\end{array}$ & $\begin{array}{l}\text { Ratio- } \\
\text { metric } \\
\Delta \lambda= \\
103,100 \\
\mathrm{~nm}\end{array}$ & 430 & $\begin{array}{l}476, \\
579 \\
\text { and } \\
468, \\
586\end{array}$ & No & $\begin{array}{l}\text { PBS (pH 7.4), } \\
1 \% \text { DMSO }\end{array}$ & $<5$ min & $\begin{array}{l}\text { HepG2 } \\
\text { cells }\end{array}$ & $\begin{array}{l}\text { No response } \\
\text { to } \mathrm{H}_{2} \mathrm{~S}, \mathrm{Cys} \text {, } \\
\mathrm{Hcy} \text { GSH; } \\
\text { response to } \\
\mathrm{HSO}_{3}{ }^{-} / \mathrm{SO}_{3}{ }^{2-}\end{array}$ \\
\hline $\begin{array}{l}\text { Sensors and } \\
\text { Actuators B, } \\
\mathbf{2 0 1 8}, \quad 254 \text {, } \\
709-718\end{array}$ & $\begin{array}{l}\text { Ratiome } \\
\text { tric } \\
\Delta \lambda= \\
117 \mathrm{~nm}\end{array}$ & 345 & $\begin{array}{l}483 \\
600\end{array}$ & No & $\begin{array}{l}\text { PBS (pH 7.4), } \\
50 \% \text { DMSO }\end{array}$ & $\begin{array}{l}\text { Within } \\
\text { sec }\end{array}$ & $\begin{array}{l}\text { MCF-7 } \\
\text { cells, } \\
\text { HeLa } \\
\text { cells, } \\
\text { Daphnia } \\
\text { magna }\end{array}$ & $\begin{array}{l}\text { No response } \\
\text { to Cys, Hcy, } \\
\text { GSH; } \\
\text { response to } \\
\mathrm{HSO}_{3}{ }^{-}\end{array}$ \\
\hline $\begin{array}{l}\text { Na-SO-LLso } \\
\text { Sensors and } \\
\text { Actuators B, } \\
2018, \quad 268, \\
157-163\end{array}$ & Turn-on & 440 & 545 & Lysosome & $\begin{array}{l}\text { pH 5.5, 5\% } \\
\mathrm{CH}_{3} \mathrm{CN}\end{array}$ & $\begin{array}{l}\text { Within } \\
\text { sec }\end{array}$ & $\begin{array}{l}\text { HeLa } \\
\text { cells }\end{array}$ & $\begin{array}{l}\text { Response to } \\
\mathrm{HSO}_{3}{ }^{-} / \mathrm{SO}_{3}{ }^{2-} \\
\text { only }\end{array}$ \\
\hline $\begin{array}{l}\text { Sensors and } \\
\text { Actuators B, } \\
2018, \quad 277, \\
576-583\end{array}$ & $\begin{array}{l}\text { Ratio- } \\
\text { metric } \\
\text { in } \\
\text { solution, } \\
\text { not in } \\
\text { cells }\end{array}$ & 460 & $\begin{array}{l}625, \\
562\end{array}$ & No & $\begin{array}{l}\text { PBS (pH 7.4), } \\
1 \% \text { DMSO }\end{array}$ & $\begin{array}{l}\text { Within } \\
\text { sec }\end{array}$ & $\begin{array}{l}\text { HeLa } \\
\text { cells }\end{array}$ & $\begin{array}{l}\text { Response to } \\
\mathrm{HSO}_{3}{ }^{-} / \mathrm{SO}_{3}{ }^{2-} \\
\text { only }\end{array}$ \\
\hline $\begin{array}{l}\text { This work } \\
\text { Tery }\end{array}$ & $\begin{array}{l}\text { Ratio- } \\
\text { metric } \\
\Delta \lambda \quad= \\
192 \mathrm{~nm}\end{array}$ & 613 & $\begin{array}{l}704, \\
512\end{array}$ & Lysosome & $\begin{array}{lrr}\text { PBS } & (\mathrm{pH} & 5), \\
30 \% & & \text { EtOH } \\
\text { with } & & 1 \% \\
\text { DMSO } & & \end{array}$ & $\begin{array}{l}\text { Within } \\
\text { sec }\end{array}$ & $\begin{array}{l}\text { HeLa } \\
\text { cells }\end{array}$ & $\begin{array}{l}\text { Response to } \\
\mathrm{HSO}_{3}{ }^{-} / \mathrm{SO}_{3}{ }^{2-} \\
\text { only }\end{array}$ \\
\hline
\end{tabular}




\section{NMR spectra}

${ }^{1} \mathrm{H} \mathrm{NMR}\left(\mathrm{CDCl}_{3}, 300 \mathrm{MHz}\right)$ of $\mathbf{1}$

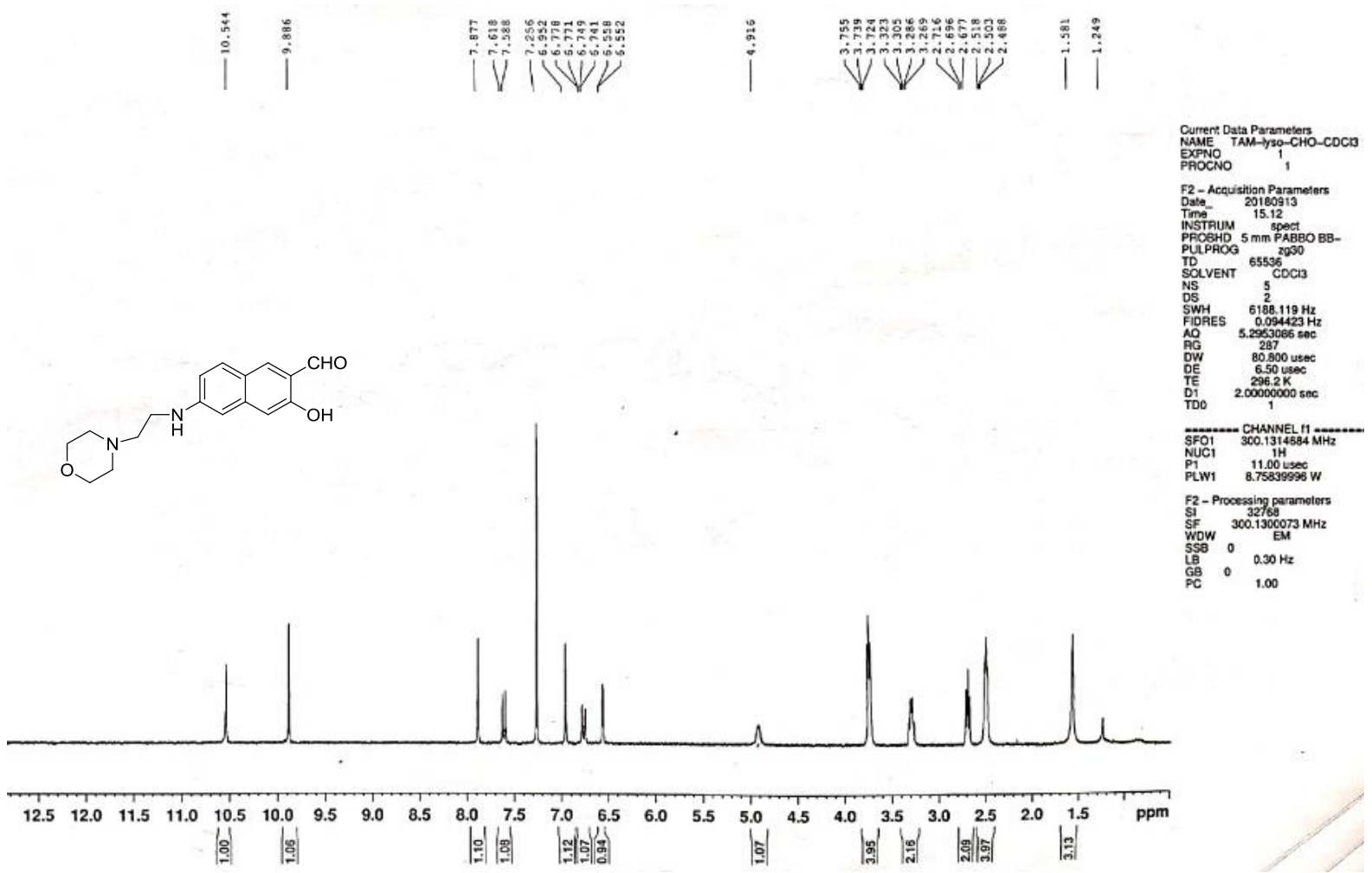

${ }^{13} \mathrm{C} \mathrm{NMR}\left(\mathrm{CDCl}_{3}, 75 \mathrm{MHz}\right)$ of 1 


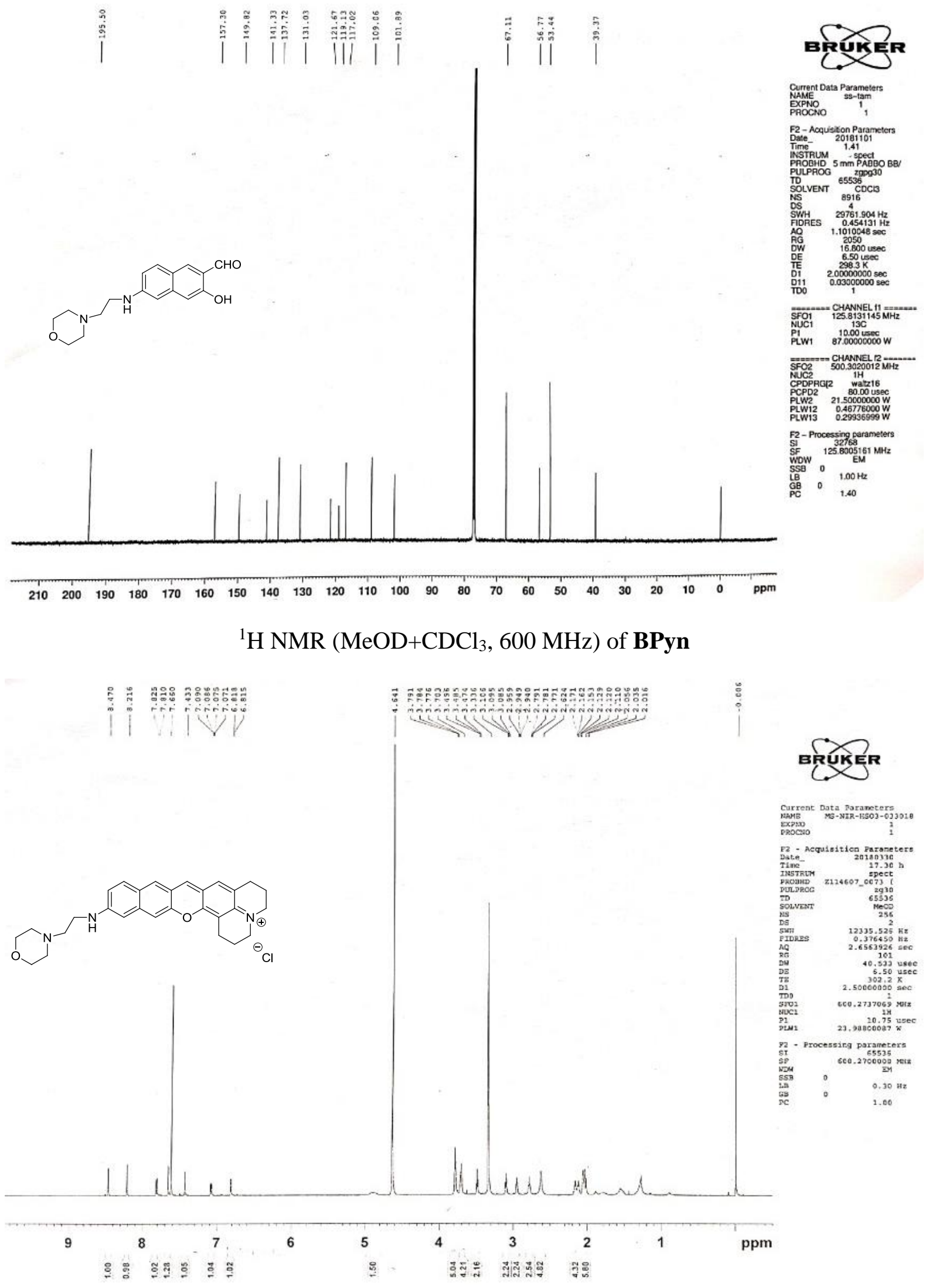




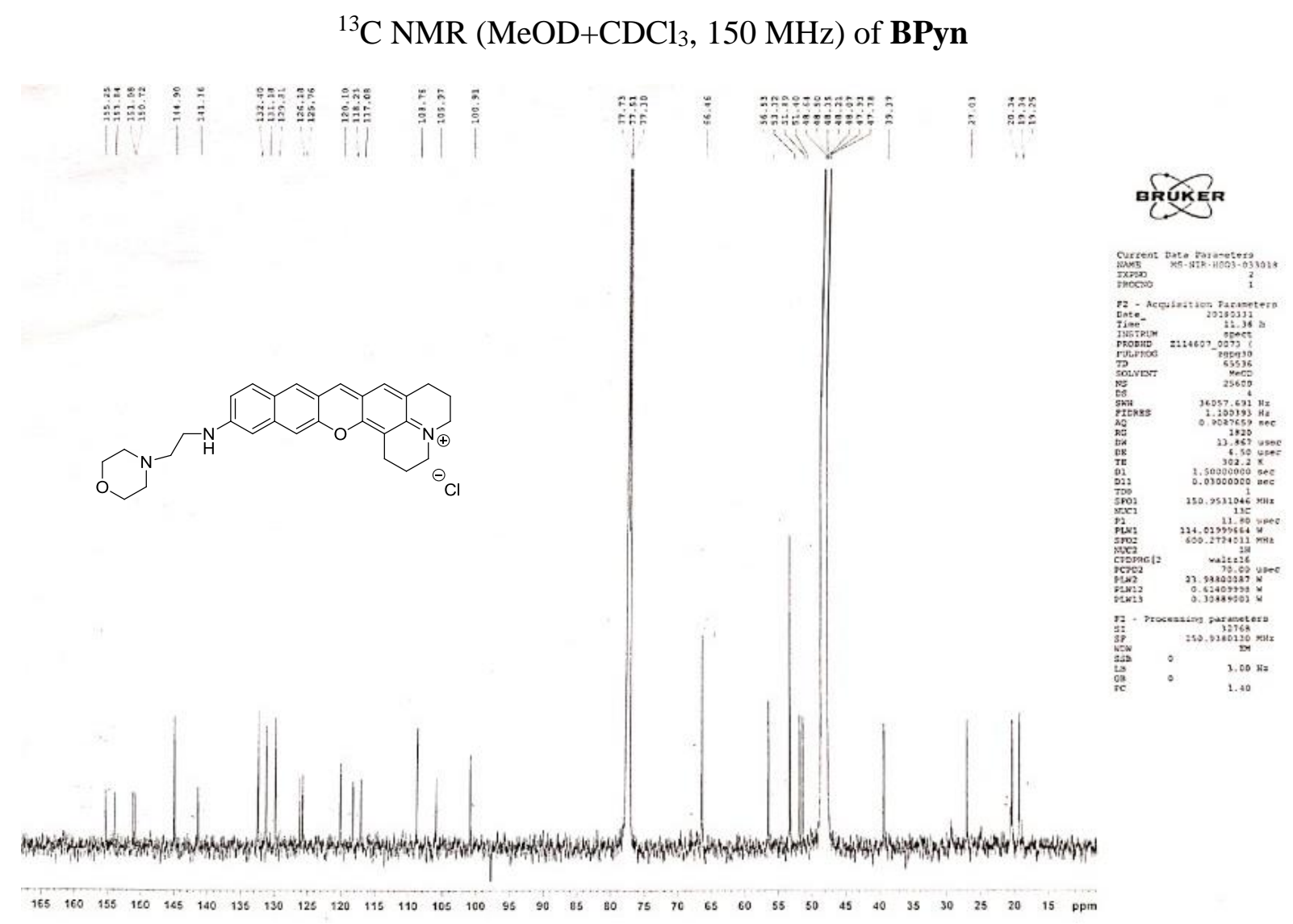

4. MASS spectra

HR-ESI(+) MS spectrum of pure BPyn 
[Mass Spectrum]

Instrument: MStation

Wample: Direct

Spectrum Type : Normal lon [EF-Linear]

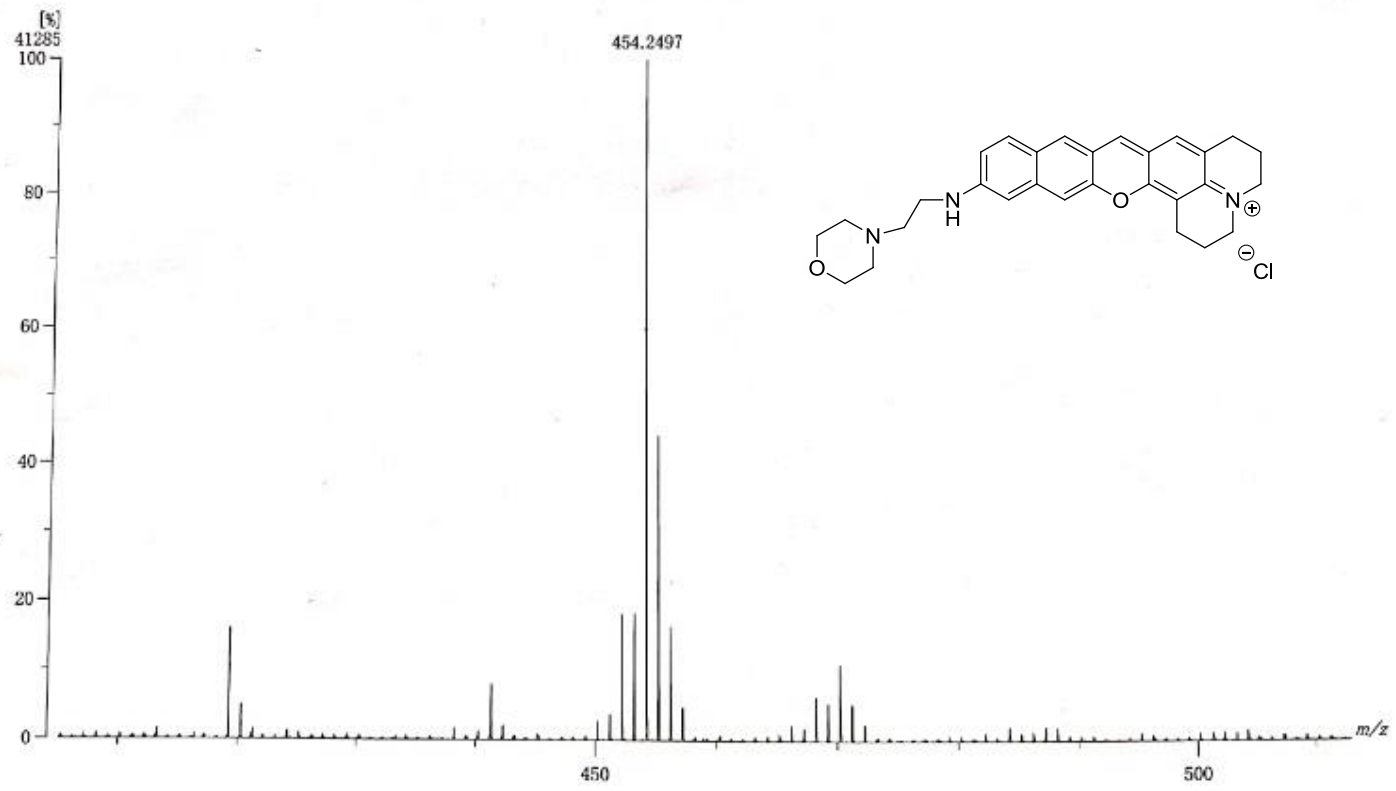

